16.

\title{
The fraction of cancer attributable to lifestyle and environmental factors in the UK in 2010
}

\section{Summary and conclusions}

\author{
DM Parkin ${ }^{*, 1}$, L Boyd ${ }^{2}$ and LC Walker ${ }^{2}$ \\ 'Centre for Cancer Prevention, Wolfson Institute of Preventive Medicine, Queen Mary University of London, Charterhouse Square, London EC IM 6BQ, UK; \\ ${ }^{2}$ Cancer Research UK, Angel Building, 407 St John Street, London ECIV 4AD, UK
}

\begin{abstract}
This chapter summarises the results of the preceding sections, which estimate the fraction of cancers occurring in the UK in 2010 that can be attributed to sub-optimal, past exposures of 14 lifestyle and environmental risk factors. For each of 18 cancer types, we present the percentage of cases attributable to one or all of the risk factors considered (tobacco, alcohol, four elements of diet (consumption of meat, fruit and vegetables, fibre, and salt), overweight, lack of physical exercise, occupation, infections, radiation (ionising and solar), use of hormones, and reproductive history (breast feeding)).

Exposure to less than optimum levels of the 14 factors was responsible for $42.7 \%$ of cancers in the UK in 2010 (45.3\% in men, $40.1 \%$ in women) - a total of about 134000 cases.

Tobacco smoking is by far the most important risk factor for cancer in the UK, responsible for 60000 cases ( $19.4 \%$ of all new cancer cases) in 2010. The relative importance of other exposures differs by sex. In men, deficient intake of fruits and vegetables (6.1\%), occupational exposures (4.9\%) and alcohol consumption (4.6\%) are next in importance, while in women, it is overweight and obesity (because of the effect on breast cancer) - responsible for $6.9 \%$ of cancers, followed by infectious agents (3.7\%).

Population-attributable fractions provide a valuable quantitative appraisal of the impact of different factors in cancer causation, and are thus helpful in prioritising cancer control strategies. However, quantifying the likely impact of preventive interventions requires rather complex scenario modelling, including specification of realistically achievable population distributions of risk factors, and the timescale of change, as well as the latent periods between exposure and outcome, and the rate of change following modification in exposure level.

British Journal of Cancer (20II) I 05, S77-S8I; doi:I0.1038/bjc.20II.489 www.bjcancer.com
\end{abstract}

(C) 20II Cancer Research UK

In this study, we have estimated the fraction of cancers occurring in the UK in 2010 that can be attributed to sub-optimal past exposures of 14 lifestyle and environmental risk factors. The optimum level of exposure or the theoretical minimum risk exposure distribution for each of the risk factors is summarised in Table 1.

Table 2 provides a summary of the percentage of cancers at each site that can be attributed to the 14 risk factors (the populationattributable fraction (PAF)). The total number of cancer cases (all sites) attributable to each risk factor was obtained by summing the numbers at the individual sites. Cases of different cancers attributable to a single risk factor are additive because each cancer case is assigned to a single ICD category.

However, cancers are caused by multiple factors acting simultaneously, and hence could be prevented by intervening on single or multiple risk factors; for example, some oesophageal cancer cases may be prevented by reducing smoking, alcohol or body weight, increasing the intake of fruits and vegetables, or by

*Correspondence: Professor DM Parkin; E-mail: d.m.parkin@qmul.ac.uk combinations of these steps. The percentages presented in Table 2 reflect the effect of removing one cause of cancer independently of other causes. But because cancers have multiple causes, the same cancers can be attributed to more than one cause, so summing the figures in the tables would overestimate the total burden of cancer attributable to the 14 risk factors. Thus, an estimate of the burden of cancer attributable to multiple causes should take into account the overlap between the effects of different carcinogens, which means that, for a specific cancer, the attributable fraction for all risk factors combined will be less than the sum of the PAFs associated with each risk factor.

When risk factors are independent (i.e., they act on different carcinogenic pathways), their effects on relative risks (RRs) will be multiplicative. This is well documented for some factors (for example, the joint effects of tobacco and alcohol), although for most there is a lack of detailed quantitative data on the risks resulting from combined exposure to several risk factors. The hypothesis of the multiplicative effect of RRs is a reasonable one, however, and allows estimation of PAFs from combined exposures. Thus, in Table 2, to obtain the last row (PAF due to all of the exposures), for each cancer, the PAF for the first exposure 
Table I Exposures considered, and theoretical optimum exposure level

\begin{tabular}{ll}
\hline Exposure & Optimum exposure level \\
\hline Tobacco smoke & Nil \\
Alcohol consumption & Nil \\
Diet & \\
$\quad$ I Deficit in intake of fruit and vegetables & $\geqslant 5$ servings ( $400 \mathrm{~g}$ ) per day \\
2 Red and preserved meat & $\geqslant 23 \mathrm{~g}$ per day \\
3 Deficit in intake of dietary fibre & $\leqslant 6 \mathrm{~g}$ per day \\
4 Excess intake of salt & $\mathrm{BMl} \leqslant 25 \mathrm{~kg} \mathrm{~m}^{-2}$ \\
Overweight and obesity & $\geqslant 30$ min 5 times per week \\
Physical exercise & Nil \\
Exogenous hormones & Nil \\
Infections & Nil \\
Radiation - ionising & As in the 1903 birth cohort \\
Radiation - solar (UV) & Nil \\
Occupational exposures & Minimum of 6 months \\
Reproduction: breast feeding &
\end{tabular}

(e.g., tobacco smoking) was subtracted from $100 \%$, and the PAF for the second exposure was applied to the remainder (the percentage not attributable to smoking). This process was performed sequentially for all relevant exposures, resulting in an estimate of the PAF for all exposures combined.

Exposure to less than optimum levels of the 14 factors was responsible for $42.7 \%$ of cancers in the UK in 2010 (45.3\% in men, $40.1 \%$ in women) - a total of about 134000 cases.

Tobacco smoking is by far the most important risk factor for cancer in the UK, responsible for 60000 cases (19.4\% of all new cancer cases) in 2010. The relative importance of other exposures differs by sex. In men, deficient intake of fruits and vegetables (6.1\%), occupational exposures (4.9\%) and alcohol consumption $(4.6 \%)$ are next in importance, while in women, it is overweight and obesity (because of the effect on breast cancer) - responsible for $6.9 \%$ of cancers, followed by infectious agents (3.7\%).

\section{SOURCES OF UNCERTAINTY}

Results are presented as the estimated percentages of different cancers attributable to specific causes in the UK population of 2010. There are several sources of uncertainty around the estimates. Some of these are quantifiable (e.g., confidence intervals of RRs and exposure prevalence, alternative choice of 'optimal exposure'), while in other cases quantification would be either very difficult (e.g., modelling lag time to provide a biologically-driven estimate of cumulative exposure) or be practically impossible (e.g., using the indirect method to estimate PAFs due to smoking).

Doll and Peto $(1981,2005)$ provided a 'range of acceptable estimates' for each exposure, to reflect the difference between those for which the risk is certain and well quantified, such as tobacco smoking, and those for which there is considerably more controversy, such as diet. We have not attempted to do so in this section; the uncertainties concerning each exposure are, however, discussed in the relevant sections. Furthermore, as we discuss below, the PAFs should not be used uncritically as a guide to the proportion of cancer cases that can be prevented by interventions.

\section{COMPARISON WITH OTHER STUDIES}

Comprehensive estimates of the fractions of cancer cases or deaths attributable to various environmental exposures have been made for world regions (Ezzati et al, 2002; Danaei et al, 2005), the United States (Danaei et al, 2009), France (IARC, 2007) and the Nordic countries (Olsen et al, 1997). For the UK, the most widely quoted are possibly those of Doll and Peto (2005), although recently the World Cancer Research Fund/American Institute for Cancer
Research published an estimate of cancers attributable to food, nutrition and physical activity in the UK and three other countries (WCRF/AICR, 2009).

The Doll and Peto (2005) estimates relate to deaths from cancer, and the methodology used is that from their 1981 monograph (Doll and Peto, 1981). The estimation method is somewhat variable for the different exposures considered. For example, they attribute to alcohol two-thirds of deaths from alcohol-related cancers (mouth, pharynx, larynx, oesophagus) in men and one-third in women, plus 'a small proportion' of liver cancer deaths. For diet, the fraction is arrived at by summing 'guestimated' fractions by which death rates of different cancers might be reduced by practical dietary means (for example: stomach $90 \%$; breast $50 \%$; cervix $20 \%)$.

The WCRF/AICR report (2009), on the other hand, uses estimates of prevalence of exposures to various nutritional factors in the UK, and estimates of RR associated with them, to calculate attributable fractions using the conventional formula. The attributable fractions so derived are generally rather greater than those estimated in this set of papers (Table 3). There are several reasons for this.

First, the WCRF/AICR estimates use current estimates of exposure prevalence applied to numbers of cancer cases in 2002 . This is unrealistic. The effects of the exposures considered are not instantaneous, and renouncing alcohol, say, would not reduce one's excess risk to zero immediately. Therefore, in the current exercise, similar to that of IARC (2007) for France, the relevant exposures are taken to be those several years earlier. This is generally 10 years, based on the follow-up periods for which most of the RRs were calculated. However, for some exposures - for example, use of post-menopausal hormones - the risk is raised in current users, but declines rapidly once exposure ceases. As most of the exposures considered have been becoming more prevalent with time, the WCRF/AICR estimates are too high for current cancer cases.

Second, the current estimates make use, whenever they are available, of dose-response summary estimates from metaanalyses by reputable authorities such as IARC, or WCRF itself, in its report 'Food, Nutrition Physical Activity and the Prevention of Cancer' (WCRF/AICR, 2007). The WCRF/AICR estimates use RR estimates from a single study, generally in a different country, to estimate the effects in the UK. This seems highly unlikely to result in a less biased result.

Finally, the current estimates use, whenever possible, per unit exposure risk estimates, and calculate attributable fractions among the proportions of the population with exposures greater or less than an acceptable 'optimum' recommended for the UK. These are dismissed by WCRF/AICR as 'associated with a number of limitations', and their estimates use RRs associated with tertiles of exposure prevalence, and estimate the effect of moving the entire UK population to the lowest tertile of exposure, defined by the study selected for the RR estimate. The baseline exposure varies, therefore, from one cancer to another; for BMI, for example, it is $<25 \mathrm{~kg} \mathrm{~m}^{-2}$ for colorectum, and $<21 \mathrm{~kg} \mathrm{~m}^{-2}$ for breast cancer.

There are a few other, perhaps more minor, points that contribute to the discrepancies: it is obviously not correct to assume all breast cancer is post-menopausal - it is only $80 \%$ of the total in UK, so that PAFs for breast cancer related to overweight/ obesity are overestimated. The same applies to PAFs for body weight and oesophagus cancer, where only the risk for adenocarcinomas is increased, and these constitute some $55 \%$ of the oesophageal cancers in the UK.

\section{SUMMARY}

Figure 1 summarises the estimates of the numbers (and percentages) of incident cancer cases in the UK in 2010 that are 


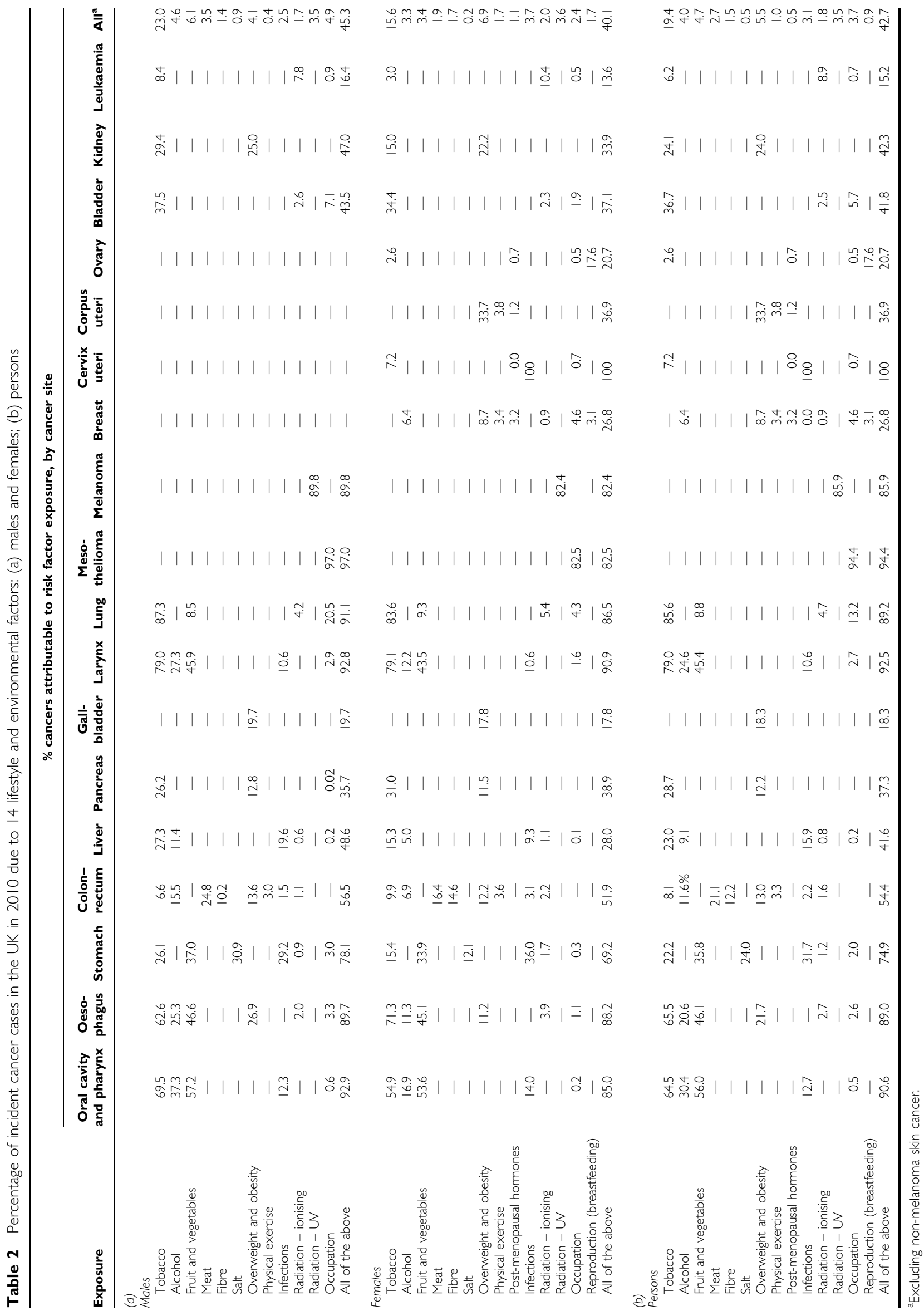


Table 3 Comparison of estimate by WCRF/AICR for the UK in 2002, with current estimate for UK 2010

\begin{tabular}{|c|c|c|c|}
\hline \multirow[b]{3}{*}{ Cancer } & \multicolumn{3}{|c|}{ Population attributable fraction (\%) } \\
\hline & \multicolumn{2}{|c|}{ WCRF/AICR (2009) } & \multirow[b]{2}{*}{ Current estimate } \\
\hline & Estimate & Range & \\
\hline \multicolumn{4}{|l|}{ Mouth, pharynx and larynx } \\
\hline Non-starchy vegetables & 34 & $(2-57)$ & 53 \\
\hline Fruits & 17 & $(0-43)$ & 53 \\
\hline Alcoholic drinks & 41 & $(4-67)$ & 29 \\
\hline Total estimate & 67 & $(0-92)$ & 67 \\
\hline \multicolumn{4}{|l|}{ Oesophagus } \\
\hline Non-starchy vegetables & 21 & $(4-40)$ & \\
\hline Fruits & 5 & $(2-9)$ & 46 \\
\hline Alcoholic drinks & 51 & $(13-74)$ & 21 \\
\hline Body fatness & 31 & $(\mid 1-49)$ & 22 \\
\hline Total estimate & 75 & $(27-93)$ & 67 \\
\hline \multicolumn{4}{|l|}{ Stomach } \\
\hline Non-starchy vegetables & 21 & $(0-4 I)$ & \\
\hline Fruits & 18 & $(3-33)$ & 36 \\
\hline Salt & 14 & $(0-39)$ & 24 \\
\hline Total estimate & 45 & $(0-76)$ & 51 \\
\hline \multicolumn{4}{|l|}{ Colon-rectum } \\
\hline Foods containing fibre & 12 & $(5-18)$ & 12 \\
\hline Red meat & 5 & $(0-21)$ & 21 \\
\hline Processed meat & 10 & $(0-23)$ & 21 \\
\hline Alcoholic drinks & 7 & $(0-18)$ & 12 \\
\hline Physical activity & 12 & $(4-20)$ & 3 \\
\hline Body fatness & 7 & $(0-17)$ & 13 \\
\hline Total estimate & 43 & $(0-73)$ & 48 \\
\hline \multicolumn{4}{|l|}{ Liver } \\
\hline Alcoholic drinks & 17 & $(0-79)$ & 9 \\
\hline \multicolumn{4}{|l|}{ Gallbladder } \\
\hline Body fatness & 16 & $(1-30)$ & 18 \\
\hline \multicolumn{4}{|l|}{ Pancreas } \\
\hline Foods containing folate ${ }^{a}$ & 23 & $(0-43)$ & \\
\hline Body fatness & 24 & $(0-43)$ & 12 \\
\hline Total estimate & 41 & $(0-67)$ & 12 \\
\hline \multicolumn{4}{|l|}{ Lung } \\
\hline Fruits & 33 & $(|7-5|)$ & 9 \\
\hline \multicolumn{4}{|l|}{ Breast } \\
\hline Alcoholic drinks & 22 & $(10-35)$ & 6 \\
\hline Physical activity & 12 & $(2-22)$ & 3 \\
\hline Body fatness & 16 & $(0-34)$ & 9 \\
\hline Total estimate & 42 & $(7-67)$ & 17 \\
\hline \multicolumn{4}{|l|}{ Endometrium } \\
\hline Physical activity & 30 & $(1 \mid-47)$ & 4 \\
\hline Body fatness & 38 & $(27-48)$ & 34 \\
\hline Total estimate & 56 & $(35-72)$ & 36 \\
\hline \multicolumn{4}{|l|}{ Prostate } \\
\hline Foods containing lycopene ${ }^{a}$ & 20 & $(0-42)$ & \\
\hline \multicolumn{4}{|l|}{ Kidney } \\
\hline Body fatness & 19 & $(12-27)$ & 24 \\
\hline All cancers ${ }^{b}$ & 26 & $(6-42)$ & 18 \\
\hline
\end{tabular}

Abbreviations: $A I C R=$ American Institute for Cancer Research; $W C R F=$ World Cancer Research Fund. ${ }^{a}$ Not evaluated in the current work. ${ }^{b}$ Excluding nonmelanoma skin cancer.

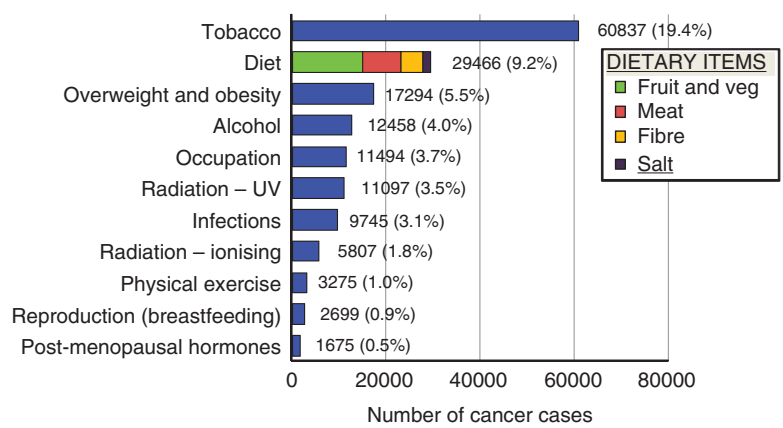

Figure I Number and percentage of cancer cases in the UK attributable to different exposures.

attributable to the 14 lifestyle and environmental exposures considered. For the most part these exposures are avoidable (ionising radiation is the exception), especially as for many (the dietary variables, physical exercise, overweight) the 'optimum' exposure represents a relatively modest recommended target. 'Avoidability' is in terms of the proportion of cancer cases that might be prevented. If the focus had been on avoidable deaths, then other interventions - especially through achieving earlier diagnosis (Richards, 2009) or generalising state-of-the-art treatment (Scottish Executive Health Department, 2001; National Audit Office, 2004) - would contribute to the total.

The four most important lifestyle exposures in Table 2 and Figure 1, tobacco smoking, dietary factors, alcohol drinking and bodyweight, account for $34 \%$ of the cancers occuring in $2010-$ almost four-fifths of the total from all 14 exposures.

It is clear that tobacco smoking remains by far the most important avoidable cause of cancer in the UK. Reducing the prevalence of smoking has been a consistent public health objective for almost 50 years since the publication of the first report on smoking and health by the Royal College of Physicians (RCP, 1962). The prevalence of cigarette smoking fell substantially in the 1970 s and the early 1980 s, from $45 \%$ in 1974 to $35 \%$ in 1982 , but the rate of decline then slowed, with prevalence falling by only about one percentage point every 2 years until 1994, after which it levelled out at about $27 \%$ before resuming a slow decline in the 2000s (Robinson and Bugler, 2010). The difference in prevalence between men and women has decreased considerably since the 1970s, and by 2008 the difference between men and women was not statistically significant, with $22 \%$ of men and $21 \%$ of women being current cigarette smokers. The overall reported number of cigarettes smoked per male and female smoker has changed little since the early 1980s. Changes in smoking-related cancer incidence lag several years behind changes in smoking prevalence, so that the current decreases in smoking-related cancer incidence and mortality will slow and eventual stop unless further progress can be achieved in reducing exposure to carcinogens in tobacco smoke.

Although it is currently not possible to pinpoint exactly what constituents of diet are protective against cancer, there is a consensus that diet is an important component of cancer risk. In the current exercise, we examine the likely impact of four components of diet for which the evidence appears to be most persuasive: fruit and vegetables and fibre (protective) and meat and salt (carcinogenic). In combination, deviation from the recommended intake levels is responsible for $9.2 \%$ of cancers in 2010 (the individual contributions are $4.7 \%$ from deficient fruit and vegetables, $2.7 \%$ from consumption of red and processed meat, $1.5 \%$ from a deficit of fibre and $0.5 \%$ from excess salt).

Excess body weight is the third most common avoidable cause of cancer in the UK, estimated to be responsible for $5.5 \%$ of 
cancers in 2010 ( $4.1 \%$ in men, $6.9 \%$ in women). In the last 15 years there have been significant increases in levels of overweight and obesity, and currently in England, a total of $66 \%$ of men and $57 \%$ of women have a BMI of $\geqslant 25 \mathrm{~kg} \mathrm{~m}^{-2}$ : this includes $22 \%$ of men and $25 \%$ of women who are obese (NHS Information Centre, 2010), defined as a BMI $>30 \mathrm{~kg} \mathrm{~m}^{-2}$. Trends among children and young people suggest that we are yet to experience the full health impact of the overweight and obesity epidemic in the UK.

Alcohol consumption is the fourth most important cause of cancer in the UK, and popular belief is that alcohol use is a highly prevalent and growing problem for the UK population. In fact, data from the national General Lifestyle Survey (Robinson and Bugler, 2010) show that the average number of units of alcohol consumed in a week rose in the 1990s to a peak in the period 2000-2002 of around 17 units for men, and 7.5 units for women, but has fallen since that time in both sexes. The proportion of men and women drinking more than the recommended maximum (21 units a week in men and 14 units in women) has also been falling. The fall in consumption occurred among men and women in all age groups, but was most evident among those aged 16-24. It is quite possible, therefore, that the burden of alcohol-related cancers is around its maximum at present, and will fall in future.
Population-attributable fractions provide a valuable quantitative appraisal of the impact of different factors in cancer causation, and are thus helpful in prioritising cancer control strategies. However, they should not be used to indicate the percentage of cancers that can currently be prevented by practical means without reference to the individual sections that discuss some of the uncertainties involved. Furthermore, quantifying the likely impact of preventive interventions requires rather complex scenario modelling, including specification of realistically achievable population distributions of risk factors, and the timescale of change, as well as the latent periods between exposure and outcome, and the rate of change following modification in exposure level (e.g., Soerjomataram et al, 2010). Thus, although $50 \%$ of colorectal cancer cases diagnosed in the UK in 2010 are attributable to lifestyle (diet, alcohol, physical inactivity and overweight), it has been estimated that only about half of this number is preventable in a reasonable $(\sim 20$-year $)$ timescale (Parkin et al, 2009).

See acknowledgements on page Si.

\section{Conflict of interest}

The authors declare no conflict of interest.

\section{REFERENCES}

Danaei G, Ding EL, Mozaffarian D, Taylor B, Rehm J, Murray CJ, Ezzati M (2009) The preventable causes of death in the United States: comparative risk assessment of lifestyle, dietary and metabolic risk factors. PLoS Med 6(4): e1000058

Danaei G, Vander Hoorn S, Lopez AD, Murray CJ, Ezzati M (2005) Causes of cancer in the world: comparative risk assessment of nine behavioural and environmental risk factors. Lancet 366: 1784-1793

Doll R, Peto R (1981) The causes of cancer: quantitative estimates of avoidable risks of cancer in the United States today. J Natl Cancer Inst 66: $1191-1308$

Doll R, Peto R (2005) Epidemiology of Cancer. In Oxford Textbook of Medicine, Warrell DA, Cox TM, Firth JD, Benz Jr EJ (eds) 4th edn. Oxford University Press (OUP): Oxford

Ezzati M, Lopez AD, Rodgers A, Vander Hoorn S, Murray CJ (2002) Selected major risk factors and global and regional burden of disease. Lancet 360: $1347-1360$

International Agency for Research on Cancer (IARC) (2007) Attributable Causes of Cancer in France in the Year 2000. IARC Working Group Reports 3. IARC: Lyon

National Audit Office (2004) Tackling Cancer in England: Saving More Lives. HMSO: London, 2004

NHS Information Centre (2010) Health Survey for England, 2009. Vol. 1 Health and Lifestyles, http://www.ic.nhs.uk/webfiles/publications/003_ Health_Lifestyles/hse09report/HSE_09_Volume1.pdf

Olsen JH, Andersen A, Dreyer L, Andersen A, Pukkala E, Tryggvadottir L, Gerhardsson de Verdier M, Winther JF (1997) Avoidable cancers in the Nordic countries. APMIS Suppl 76: 1-146

Parkin DM, Olsen AH, Sasieni P (2009) The potential for prevention of colorectal cancer in the UK. Eur J Cancer Prev 18: 179-190

Richards MA (2009) The size of the prize for earlier diagnosis of cancer in England. Br J Cancer 101(Suppl 2): S125-S129

Robinson S, Bugler C (2010) Smoking and Drinking Among Adults. Office of National Statistics, 2010. http:/www.ons.gov.uk/ons/rel/ghs/general-lifestylesurvey/2008-report/smoking-and-drinking-among-adults- -2008.pdf

Royal College of Physicians (1962) Smoking and Health: A Report of the Royal College of Physicians on Smoking in Relation to Cancer of the Lung and Other Diseases. RCP: London

Scottish Executive Health Department. Cancer Scenarios: An Aid to Planning Cancer Services in Scotland in the Next Decade. The Scottish Executive: Edinburgh, (2001)

Soerjomataram I, de Vries E, Engholm G, Paludan-Müller G, BrønnumHansen H, Storm HH, Barendregt JJ (2010) Impact of a smoking and alcohol intervention programme on lung and breast cancer incidence in Denmark: an example of dynamic modeling with Prevent. Eur J Cancer 46: $2617-2624$

World Cancer Research Fund (WCRF)/American Institute for Cancer Research (AICR) (2007) Food, Nutrition, Physical Activity, and the Prevention of Cancer: a Global Perspective. AICR: Washington, DC

World Cancer Research Fund (WCRF)/American Institute for Cancer Research (AICR) (2009) Policy and Action for Cancer Prevention. Food, Nutrition and Physical Activity: A Global Perspective. AICR: Washington, DC

cC)(2) This work is licensed under the Creative Commons Attribution-NonCommercial-Share Alike 3.0 Unported License. To view a copy of this license, visit http://creativecommons. org/licenses/by-nc-sa/3.0/ 